\title{
Molecular determination of Enterocytozoon bieneusi in pet shop puppies and breeding kennel dogs
}

\author{
Totsapon Phrompraphai ${ }^{1}$, Naoyuki Itoh ${ }^{1 *}$, Yuko Iijima ${ }^{1}$, Yoichi Ito ${ }^{1,2}$, Yuya Kimura ${ }^{1}$ and Satoshi Kameshima ${ }^{1}$ \\ ${ }^{1}$ Laboratory of Small Animal Internal Medicine, School of Veterinary Medicine, Kitasato University, Higashi 23-35-1, Towada, Aomori 034-8628, Japan \\ ${ }^{2}$ Ito Animal Hospital, Gakuendai 2-2-1, Miyashiro, Minami Saitama, Saitama 345-0826, Japan
}

\begin{abstract}
Enterocytozoon (E.) bieneusi is the most common opportunistic pathogen in humans with immunocompromised status. This microorganism has been reported in various mammals including humans and companion animals. There are at least 90 genotypes of E. bieneusi, and some of them are considered zoonotic. Although pet shops and breeding kennels are the major source of dogs for owners, only a few reports are available regarding the molecular determination of $E$. bieneusi in pet shop puppies, and there are no reports in breeding kennels dogs. The present study reports the molecular prevalence and genotypes of $E$. bieneusi in 621 pet shop puppies and 314 breeding kennel dogs in Japan. The overall prevalences of E. bieneusi infection were 6.1 and $11.8 \%$ in pet shop puppies and breeding kennel dogs, respectively. The prevalences are significantly different depending on facilities, and appointed facilities maintained high levels of E. bieneusi infection. Due to the concentrated environmental contamination and the stressful situation, which can induce an immunosuppressive status, the reinfection and/or the reactivation of microsporidiosis are likely to be the major reasons for the high levels of infection in some facilities. Regarding the sequencing data of 75 polymerase chain reaction (PCR)-positive samples, 70 isolates correspond to E. bieneusi genotype PtEb IX and the remaining five are identified as genotype CD7. The importance of pet shop puppies and breeding kennel dogs as reservoirs for E. bieneusi transmission to humans is likely to be low in Japan, since both isolated genotypes here are recognized as canine specific.
\end{abstract}

Enterocytozoon (E.) bieneusi is most commonly determined worldwide as an opportunistic pathogen in symptomatic (chronic diarrhea) human patients with immunocompromised status due to factors, such as acquired immunodeficiency syndrome (AIDS), organ transplant, and malignant diseases [1-3]. Although the accurate transmission routes and origins for human infections are poorly understood, the spore of E. bieneusi are shed into the feces of the infected host, and the new host can infect via the ingestion of environmental spores in contaminated water or food [2-4]. This microorganism has been reported in various mammals, including humans, wild, domestic, and companion animals [5,6]. In addition, recent molecular approaches have demonstrated that E. bieneusi has more than 90 genotypes, and some of them are considered zoonotic genotypes because of the determination in both humans and animals [2,5]. Therefore, animals have the potential to act as a reservoir of E. bieneusi transmission to humans. Dogs are likely the most important reservoir due to their close contact with humans. Epidemiological reports of $E$. bieneusi infection in dogs are increasing [7-11]. Considering the scale of research, however, only a few reports are available regarding the molecular determination of E. bieneusi in dogs from pet shops, which is the major source of puppies for private owners [10]. Furthermore, there is no report on the molecular detection of E. bieneusi in dogs of breeding kennels that are occupied the upper stream for pet shops as the place of puppy reproduction. The purpose of the present study was to investigate the molecular prevalence and was to characterize the genotypes of E. bieneusi in pet shop puppies and breeding kennel dogs in Japan.

A total of 621 and 314 fresh fecal samples were randomly collected on a single occasion from pet shop puppies ( $\leq 3$ months old) and breeding kennel dogs (from 2 months old to 11 years old and divided into two groups: $<1$ year old vs. $\geq 1$ year old), respectively, between
August 2014 and July 2017. The pet shops included four pet shops located in three different prefectures (Aomori: 2 pet shops; PS-A and B, Saitama: 1 pet shop; PS-C, Ibaraki: 1 pet shop; PS-D) in east Japan. The breeding kennels consisted five breeding kennels located in five different prefectures (Miyagi: 1 breeding kennel; BK-1, Niigata: 1 breeding kennel; BK-2, Gunma: 1 breeding kennel; BK-3, Shizuoka: 1 breeding kennel; BK-4, Aichi: 1 breeding kennel; BK-5) in Japan. All fecal samples were naturally defecated, and were donated by the managers, who granted permission to include their dogs in the examination. After the evaluation of fecal condition (formed vs. soft vs. diarrhea), the spores of E. bieneusi were recovered using a sucrose gradient concentration method with a specific gravity of 1.26 . The DNA extraction was performed using a QIAamp DNA Mini Kit (QIAGEN GmbH, Hilden, Germany) according to the manufacturer's instructions. The obtained DNA samples were stored at $-20^{\circ} \mathrm{C}$ prior to analysis.

Anested polymerasechain reaction(PCR) assaytargeting theinternal transcribed spacer (ITS) region of ribosomal DNA was applied to the detection of E. bieneusi. In the primary reaction, forward primer EBITS3 (5' -GGTCATAGGGATGAAGAG-3') and reverse primer EBITS4 (5'-TTCGAGTTCTTTCGCGCTC-3') were used for amplification of

*Correspondence to: Naoyuki Itoh, Laboratory of Small Animal Internal Medicine, School of Veterinary Medicine, Kitasato University, Higashi 23-35-1, Towada, Aomori 034-8628, Japan, Tel: 81176249440; Fax: 81176249440; E-mail: naoitoh@vmas.kitasato-u.ac.jp

Key words: dog, Enterocytozoon bieneusi, pet shop, breeding kennel, puppy, genotype

Received: October 21, 2018; Accepted: November 09, 2018; Published: November 12, 2018 
an approximately $435 \mathrm{bp}$ DNA fragment. In the secondary reaction, forward primer EBITS1 (5'-GCTCTGAATATCTATGGCT-3') and reverse primer EBITS2.4 (5'-ATCGCCGACGGATCCAAGTG-3') were used to amplify an approximately $390 \mathrm{bp}$ fragment [12]. For the primary reaction, the PCR mixture was composed $1 \times$ buffer containing $1.5 \mathrm{mM}$ of $\mathrm{MgCl}_{2}, 200 \mu \mathrm{M}$ of each dNTP, $0.5 \mu \mathrm{M}$ of each primer, 1.25 units of GoTaq DNA polymerase (Promega Corporation, Madison, WI, USA), and $3.0 \mu \mathrm{l}$ of template DNA in a total reaction volume of 25 $\mu$ l. For the secondary reaction, the PCR mixture was the same as that for the primary reaction, with the exception of primary PCR amplicons, which were used as a template. The following cycling parameters were used for the primary reaction: after an initial denaturation of $3 \mathrm{~min}$ at $94^{\circ} \mathrm{C}, 35$ cycles were performed, each consisting of $30 \mathrm{sec}$ at $94^{\circ} \mathrm{C}$ for denaturation, $30 \mathrm{sec}$ at $57^{\circ} \mathrm{C}$ for annealing, and $40 \mathrm{sec}$ at $72^{\circ} \mathrm{C}$ for extension, with a final extension of $10 \mathrm{~min}$ at $72^{\circ} \mathrm{C}$. For the secondary reaction, the parameters were the same as those for the primary reaction, except that the annealing temperature was $55^{\circ} \mathrm{C}$.

All secondary PCR products were examined by electrophoresis on $1.5 \%$ agarose gels. The specific DNA fragments (approximately $390 \mathrm{bp}$ ) were confirmed after alternative ethidium bromide staining under ultraviolet (UV) light using a transilluminator. Secondary PCR amplicons were purified using a QIAquick Gel Extraction kit (QIAGEN $\mathrm{GmbH}$, Hilden, Germany) and sequenced with the secondary primer set. Sequences were analyzed by a commercial laboratory (FASMAC Co., Ltd., Atsugi, Kanagawa, Japan). Sequence alignment and compilation were performed using the MEGA 6.06 (www. megasoftware.net) program. To determine the genotypes of $E$. bieneusi, the DNA sequences were compared to GenBank references by BLAST (Basic Local Alignment Search Tool) searches (http://www.ncbi.nlm. nih.gov/), and their similarity was determined based on the degree of sequence identity. Data were analyzed statistically using Fisher's exact probability test, with values of $P<0.05$ considered significant.

As determined by conventional PCR, we could confirm the positive samples for E. bieneusi infection via the specific DNA fragments at the position of approximately $390 \mathrm{bp}$ (Figure 1). Overall, E. bieneusi infection was positive in 38 animals (6.1\%) of the 621-pet shop puppies and was determined in all facilities (Table 1). The prevalence of each pet shops ranged from 1.3 to $12.2 \%$, and the highest prevalence of PS-D (12.2\%) was statistically significant in comparison to the other three pet shops (PS-A, PS-B, and PS-C). In 314 breeding kennel dogs, overall prevalence of E. bieneusi infection was $11.8 \%$, which was significantly $(P<0.01)$ higher than that of pet shop puppies. As in the pet shop puppies, E. bieneusi infection was confirmed in all breeding kennels ranging from 2.1 to $20.3 \%$. The prevalence of BK-5 (20.3\%) was significantly higher than those of three breeding kennels (BK-1, BK-2, and BK-3). In addition, although there was no statistical significance, the prevalence in $<1$-year-old breeding kennel dogs $(22.5 \%)$ revealed a higher tendency than that of $\geq 1$-year-old dogs (10.2\%). No significant differences were observed in fecal condition in both pet shop puppies and breeding kennel dogs.

A sequencing analysis of the ITS region of ribosomal DNA fragments demonstrated that 37 of 38 PCR-positive samples from the pet shop puppies shared 99 to $100 \%$ similarity with the sequences of E. bieneusi genotype PtEb IX (accession number KJ668719) retrieved from the GenBank database (Table 2). Only one sample from PS-A corresponded to genotype CD7 (accession number KJ668734) with $100 \%$ similarity. In the breeding kennel dogs, 33 isolates of 37 PCRpositive samples were genotype PtEb IX (accession number KJ668719), with 99 to $100 \%$ similarity, and the remaining 4 isolates from two
Table 1. Molecular determination of Enterocytozoon bieneusi in pet shop puppies and breeding kennel dogs

\begin{tabular}{|c|c|c|c|c|}
\hline & Examined & Positives & Prevalence (\%) & $P$-value \\
\hline \multicolumn{5}{|c|}{ Pet shop puppies } \\
\hline Overall & 621 & 38 & 6.1 & - \\
\hline \multicolumn{5}{|c|}{ Fecal conditions } \\
\hline Formed & 550 & 32 & 5.8 & N.S. \\
\hline Soft & 53 & 6 & 11.3 & - \\
\hline Diarrhea & 18 & 0 & 0 & N.S. \\
\hline \multicolumn{5}{|l|}{ Pet shops } \\
\hline PS-A & 235 & 13 & 5.5 & $<0.05$ \\
\hline PS-B & 153 & 5 & 3.3 & $<0.01$ \\
\hline PS-C & 77 & 1 & 1.3 & $<0.01$ \\
\hline PS-D & 156 & 19 & 12.2 & - \\
\hline \multicolumn{5}{|c|}{ Breeding kennel dogs } \\
\hline Overall & 314 & 37 & 11.8 & - \\
\hline \multicolumn{5}{|l|}{ Age } \\
\hline$<1$-year-old & 40 & 9 & 22.5 & - \\
\hline$\geq 1$-year-old & 274 & 28 & 10.2 & N.S. \\
\hline \multicolumn{5}{|c|}{ Fecal conditions } \\
\hline Formed & 271 & 36 & 13.3 & - \\
\hline Soft & 41 & 1 & 2.4 & N.S. \\
\hline Diarrhea & 2 & 0 & 0 & N.S. \\
\hline \multicolumn{5}{|c|}{ Breeding kennels } \\
\hline BK-1 & 48 & 1 & $2.1^{*}$ & $<0.01$ \\
\hline BK-2 & 87 & 4 & $4.6^{*}$ & $<0.01$ \\
\hline BK-3 & 25 & 1 & 4.0 & N.S. \\
\hline BK-4 & 31 & 6 & 19.4 & N.S. \\
\hline BK-5 & 123 & 25 & 20.3 & - \\
\hline
\end{tabular}

"The parameters were also significant differences $(P<0.05)$ when compared with BK-4

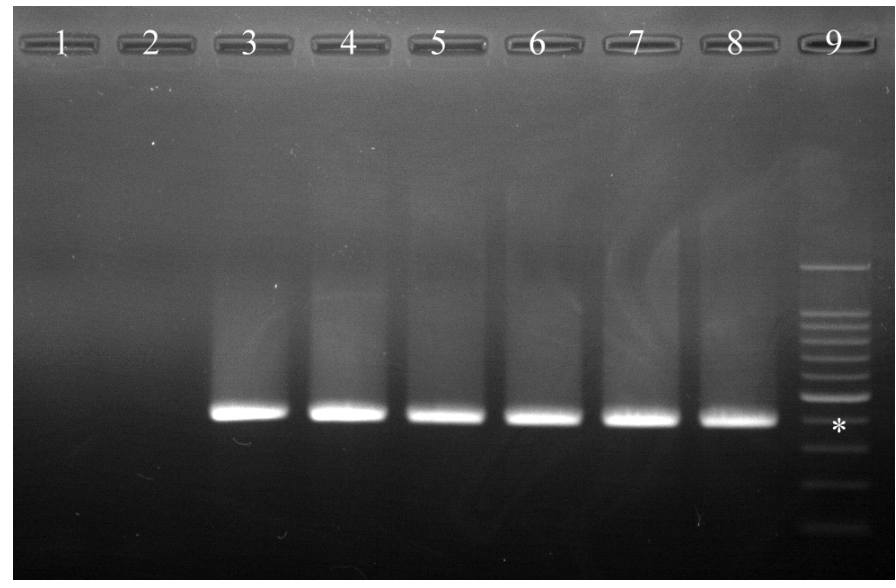

Figure 1. PCR products on 1.5\% agarose gel. Lane 1-2: Enterocytozoon bieneusi negative control, Lane 3: E. bieneusi positive control, Lane 4: E. bieneusi genotype PtEb IX from pet shop (PS-B), Lane 5: E. bieneusi genotype PtEb IX from breeding kennel (BK-4), Lane 6: E. bieneusi genotype CD7 from pet shop (PS-A), Lane 7: E. bieneusi genotype CD7 from breeding kennel (BK-2), Lane 8: E. bieneusi genotype CD7 from breeding kennel (BK-5), Lane 9: 100 bp DNA ladder. "Indicates approximately 390 bp

breeding kennels (BK-2 and BK-5) corresponded to genotype CD7 (accession number KJ668734) with 99-100\% similarity (Figure 2).

We began the present study with the hypothesis that the dogs kept in pet shops and breeding kennels indicated the markedly high prevalence of E. bieneusi infection, likely due to the intestinal protozoan Giardia and Cryptosporidium [13-17]. Contrary to our expectation, the present study demonstrated the low levels of E. bieneusi infection, such as the overall prevalences of 6.1 and $11.8 \%$ in pet shop puppies and breeding 


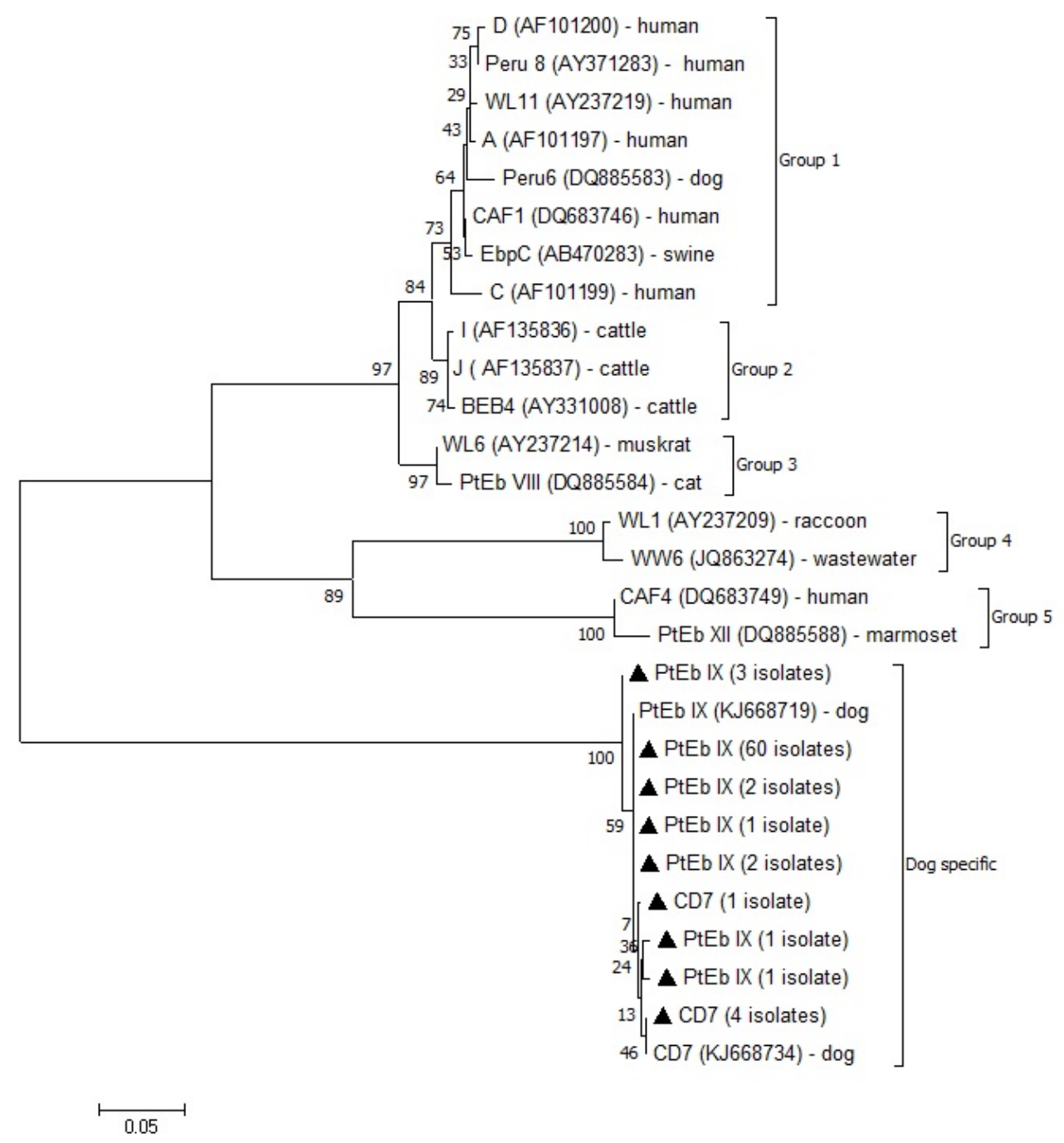

Figure 2. Phylogenetic analysis of ITS nucleotide sequences of Enterocytozoon bieneusi genotypes isolated from pet shop puppies and breeding kennel dogs in the present study $\boldsymbol{\Delta}=$ Isolates from dogs in the present study

Table 2. Enterocytozoon bieneusi genotypes in pet shop puppies and breeding kennel dogs

\begin{tabular}{|c|c|}
\hline \multicolumn{2}{|c|}{ genotypes } \\
\hline \multicolumn{2}{|c|}{ Pet shop puppies } \\
\hline Pet shops & PtEb IX (12)*, CD7 (1) \\
\hline PS-A & PtEb IX (5) \\
\hline PS-B & PtEb IX (1) \\
\hline PS-C & PtEb IX (19) \\
\hline PS-D & \\
\hline \multicolumn{2}{|c|}{ Breeding kennel dogs } \\
\hline Breeding kennels & PtEb IX (1) \\
\hline BK-1 & PtEb IX (3), CD7 (1) \\
\hline BK-2 & PtEb IX (1) \\
\hline BK-3 & PtEb IX (6) \\
\hline BK-4 & PtEb IX (22), CD7 (3) \\
\hline BK-5 &
\end{tabular}

kennel dogs, respectively. Regarding dogs kept in pet shops and pet markets, approximately the same level molecular prevalence levels have been reported in China: 7.7\% (2/26) [7], 14.8\% (16/108) [9], and 5.9\% $(19 / 322)$ [10]. The current prevalence recorded in breeding kennel dogs is impossible to compare due to the lack of previous data from breeding kennels. However, in pet shop puppies, the overall prevalence of each pet shop ranged from 1.3 to $12.2 \%$, and only one shop had a prevalence (PS-D; 12.2\%) significantly higher than others. Moreover, in breeding kennels, including young dogs as well as adult animals, two facilities of BK-4 (19.4\%) and BK-5(20.3\%) recorded higher prevalences than others (2.1-4.6\%). The lower prevalence determined here in some pet shops and breeding kennels were the same as the level recorded previously in private household dogs of $4.9 \%$ (4/82) in Poland [11], and $1.4 \%(2 / 141)$ in China [9]. It is well demonstrated that the immune response is important to control the opportunistic Microsporidia infections. The immature and suppressive status of immune systems is a risk factor for microsporidiosis [1-4,18]. Therefore, younger dogs are at high risk due to their immature immune systems $[19,20]$. However, the present results suggest that the prevalence of $E$. bieneusi infection is affected by the condition of the facility rather than the age of dogs because the prevalences of pet shop puppies and breeding kennel dogs are significantly different depending on facilities and appointed facilities maintained high levels of $E$. bieneusi infections. In addition, the prevalence of adult dogs kept in breeding kennels $(10.2 \%)$ is significantly $(P<0.05)$ higher than that of pet shop puppies $(6.1 \%)$. Microsporidia spores are resistant in environments for extended time 
periods; therefore, there is a potential for infection from environmental contamination $[1,3]$. Contaminated water is one of the primary causes of human microsporidiosis [2-4]. We did not evaluate the sanitary management and environmental condition in each facility. However, the reinfection and/or the reactivation of microsporidiosis according to the concentrated environmental contamination by E. bieneusi spores and the frequent close contact with other dogs in the limited space, which is a stressful situation for animals and can induce the immunosuppressive status, are likely to be major causes of high levels of infection in some facilities $[4,17,18,21,22]$. The results of the present study suggest that there is no correlation between E. bieneusi infection and fecal condition disorder (soft or diarrhea). Despite the fact that the etiology and pathogenicity of Microsporidia have not been clarified, the cases of asymptomatic spore shedding are recognized in dogs and humans, including both immunocompromised and immunocompetent individuals $[2,4,9,23]$. The immunosuppressed trigger, for example human immunodeficiency virus (HIV) infection, is also indicated to contributes to the symptomatic infections $[2,18,24,25]$.

The present sequencing data of PCR-positive samples demonstrates the dominancy of E. bieneusi genotype PtEb IX in pet shop puppies and breeding kennel dogs in Japan, because this genotype was isolated from all facilities of pet shops and breeding kennels. It is easy to understand this result, because the genotype PtEb IX is recognized as a caninespecific genotype and its isolation is restricted to dogs worldwide, except for a few rare cases [8-11,26,27]. Five isolates of the E. bieneusi genotype $\mathrm{CD} 7$, which derive from one pet shop (PS-A) and two breeding kennels (BK-2 and BK-5), have been determined here for first time in Japan. Genotype CD7 has recently been described in China as the non-dominant canine-specific genotype, although it has not been reported in other countries [8,9]. The results suggest that at least two canine-specific E. bieneusi genotypes invade dogs in Japan, and the importance of pet shop puppies and breeding kennel dogs as reservoirs for E. bieneusi transmission to humans is likely to be low in Japan.

\section{Conflicts of interest}

On behalf of all authors, the corresponding author states that there is no conflict interest.

\section{References}

1. Didier ES, Weiss LM (2006) Microsporidiosis: Current status. Curr Opin Infect Dis 19: 485-492. [Crossref]

2. Matos O, Lobo ML, Xiao L (2012) Epidemiology of Enterocytozoon bieneusi infection in humans. J Parasitol Res 2012: 981424. [Crossref]

3. Han B, Weiss LM (2017) Microsporidia: obligate intracellular pathogens within the fungal kingdom. Microbiol Spectr 5. [Crossref]

4. Didier ES, Weiss LM (2011) Microsporidiosis: not just in AIDS patients. Curr Opin Infect Dis 24: 490-495. [Crossref]

5. Santín M, Fayer R (2011) Microsporidiosis: Enterocytozoon bieneusi in domesticated and wild animals. Res Vet Sci 90: 363-371. [Crossref]

6. Wang SS, Wang RJ, Fan XC, Liu TL, Zhang LX, et al. (2018) Prevalence and genotypes of Enterocytozoon bieneusi in China. Acta Trop 183: 142-152. [Crossref]
7. Zhang X, Wang Z, Su Y, Liang X, Sun X, et al. (2011) Identification and genotyping of Enterocytozoon bieneusi in China. J Clin Microbiol 49: 2006-2008. [Crossref]

8. Karim MR, Dong H, Yu F, Jian F, Zhang L, et al. (2014) Genetic diversity in Enterocytozoon bieneusi isolates from dogs and cats in China: host specificity and public health implications. J Clin Microbiol 52: 3297-3302. [Crossref]

9. Li W, Li Y, Song M, Lu Y, Yang J, et al. (2015) Prevalence and genetic characteristic of Cryptosporidium, Enterocytozoon bieneusi and Giardia duodenalis in cats and dogs in Heilongjing province, China. Vet Parasitol 208: 125-134. [Crossref]

10. Xu H, Jin Y, Wu W, Li P, Wang L, et al. (2016) Genotypes of Cryptosporidium spp., Enterocytozoon bieneusi and Giardia duodenalis in dogs and cats in Shanghai, China. Parasit Vectors 9: 121. [Crossref]

11. Piekarska J, Kicia M, Wesołowska M, Kopacz Ż, Gorczykowski M, et al. (2017) Zoonotic microsporidia in dogs and cats in Poland. Vet Parasitol 246: 108-111. [Crossref]

12. Reetz J, Nöckler K, Reckinger S, Vargas MM, Weiske W, et al. (2009) Identification of Encephalitozoon cuniculi genotype III and two novel genotypes of Enterocytozoon bieneusi in swine. Parasitol Int 58: 285-292. [Crossref]

13. Giangaspero A, Iorio R, Paoletti B, Traversa D, Capelli G (2006) Molecular evidence for Cryptosporidium infection in dogs in central Italy. Parasitol Res 99: 297-299. [Crossref]

14. Itoh N, Itagaki T, Kawabata T, Konaka T, Muraoka N, et al. (2011) Prevalence of intestinal parasites and genotyping of Giardia intestinalis in pet shop puppies in east Japan. Vet Parasitol 176: 74-78. [Crossref]

15. Itoh N, Oohashi Y, Ichikawa-Seki M, Itagaki T, Ito Y, et al. (2014) Molecular detection and characterization of Cryptosporidium species in household dogs, pet shop puppies, and dogs kept in a school of veterinary nursing in Japan. Vet Parasitol 200: 284-288. [Crossref]

16. Itoh N, Kanai K, Kimura Y, Chikazawa S, Hori Y, et al. (2015) Prevalence of intestinal parasites in breeding kennel dogs in Japan. Parasitol Res 114: 1221-1224. [Crossref]

17. Kim R (2018) Effects of surgery and anesthetic choice on immunosuppression and cancer recurrence. J Transl Med 16: 8. [Crossref]

18. Moretto MM, Harrow DI, Khan IA (2015) Effector CD8 T cell immunity in microsporidial infection: a lone defense mechanism. Semin Immunopathol 37: 281287. [Crossref]

19. Faldyna M, Levá L, Knötigová P, Toman M (2001) Lymphocyte subsets in peripheral blood of dogs-a flow cytometric study. Vet Immunol Immunopathol 82: 23-37. [Crossref]

20. Day MJ (2007) Immune system development in the dog and cat. J Comp Pathol 137 Suppl 1: S10-15. [Crossref]

21. Mastorakos G, Karoutsou EI, Mizamtsidi M (2006) Corticotropin releasing hormone and the immune/inflammatory response. Eur J Endocrinol 155: S77-S84. [Crossref]

22. Powell ND, Allen RG, Hufnagle AR, Sheridan JF, Bailey MT (2011) Stressor-induced alterations of adaptive immunity to vaccination and viral pathogens. Immunol Allergy Clin N Am 31: 69-79. [Crossref]

23. Jamshidi Sh, Tabrizi AS, Bahrami M, Momtaz H (2012) Microsporidia in household dogs and cats in Iran; a zoonotic concern. Vet Parasitol 185: 121-123. [Crossref]

24. Ghosh K, Weiss LM (2012) T cell response and persistence of the microsporidia. FEMS Microbiol Rev 36: 748-760. [Crossref]

25. Valencakova A, Halanova M (2012) Immune response to Encephalitozoon infection review. Comp Immunol Microbiol Infect Dis 35: 1-7. [Crossref]

26. Santín M, Cortés Vecino JA, Fayer R (2008) Enterocytozoon bieneusi genotypes in dogs in Bogota, Colombia. Am J Trop Med Hyg 79: 215-217. [Crossref]

27. Abe N, Kimata I, Iseki M (2009) Molecular evidence of Enterocytozoon bieneusi in Japan. J Vet Med Sci 71: 217-219. [Crossref]

Copyright: (C2018 Phrompraphai T. This is an open-access article distributed under the terms of the Creative Commons Attribution License, which permits unrestricted use, distribution, and reproduction in any medium, provided the original author and source are credited. 